\title{
Efeitos das Fontes e Níveis de Sódio, Cloro e Potássio e da Relação (Na + K)/Cl, sobre o Desempenho e Características do Plasma Sanguíneo de Poedeiras Comerciais ${ }^{1}$
}

\author{
Otto Mack Junqueira², Benedito de Camargo Filho ${ }^{3}$, Lúcio Francelino Araújo ${ }^{4}$, \\ Cristiane Soares da Silva Araújo ${ }^{4}$, Nilva Kazue Sakomura²
}

\begin{abstract}
RESUMO - Dois experimentos foram realizados para estudar os efeitos da relação $(\mathrm{Na}+\mathrm{K}) / \mathrm{Cl}$ na dieta de poedeiras comerciais no final do ciclo de postura, pela adição de bicarbonato de sódio $\left(\mathrm{NaHCO}_{3}\right)$, cloreto de sódio $(\mathrm{NaCl})$, cloreto de potássio $(\mathrm{KCl})$ e cloreto de amônia $\left(\mathrm{NH}_{4} \mathrm{Cl}\right)$, avaliando-se os parâmetros produtivos e características do plasma sangüíneo. O primeiro experimento envolveu cinco tratamentos: $0,30 \%$ de NaCl$; 0,30 \%$ de $\mathrm{NaCl}+0,95 \%$ de $\mathrm{NaHCO}_{3} ; 0,38 \%$ de $\mathrm{KCl}+0,67 \%$ de $\mathrm{NaHCO}_{3} ; 0,38 \%$ de $\mathrm{KCl}+1,62 \%$ de $\mathrm{NaHCO}$; e $0,38 \%$ de $\mathrm{KCL}+2,56 \%$ de $\mathrm{NaHCO}_{3}$. A relação $(\mathrm{Na}+\mathrm{K}) / \mathrm{Cl}$ foi, respectivamente, 3,46; 4,46; 4,46; 5,46; e 6,46. O segundo experimento envolveu quatro tratamentos: $0,30 \% \mathrm{NaCl} ; 0,47 \%$ de $\mathrm{NaHCO}_{3}+0,19$ de $\mathrm{NH}_{4} \mathrm{Cl} ; 1,17 \% \mathrm{NaHCO}_{3}+0,19 \%$ de $\mathrm{NH}_{4} \mathrm{Cl} ; \mathrm{e} 1,88 \%$ de $\mathrm{NaHCO}$ $+0,19 \%$ de $\mathrm{NH}_{4} \mathrm{Cl}$. A relação $(\mathrm{Na}+\mathrm{K}) / \mathrm{Cl}$ foi 3,46; 4,46; 5,46; e 6,46, respectivamente. No primeiro experimento, o maior consumo alimentar foi observado nas aves alimentadas com dietas contendo $0,30 \%$ de $\mathrm{NaCl}$. As aves que consumiram $\mathrm{KCl}$ apresentaram melhor conversão alimentar. Foi observado aumento no fósforo e pH do plasma sangüíneo. Aves que receberam dietas em que a relação $(\mathrm{Na}+\mathrm{K}) / \mathrm{Cl}$ foi de 5,46 apresentaram maiores níveis de fósforo no plasma sangüíneo. O aumento do $\mathrm{pH}$ no plasma sangüíneo foi observado em aves que receberam dietas com relações de $(\mathrm{Na}+\mathrm{K}) / \mathrm{Cl}$ de 4,46 e 6,46. No segundo experimento, o peso do ovo diminuiu com o aumento do $\mathrm{NaHCO}_{3}$ da dieta.
\end{abstract}

Palavras-chave: características plasmáticas, desempenho, parâmetros sangüíneos, poedeiras, qualidade da casca do ovo

\section{Effects of the Source and Levels of Sodium, Chlorine and Potassium and $(\mathrm{Na}+\mathrm{K}) / \mathrm{Cl}$ Ratio on Performance and Plasma Blood Characteristics of Laying Hens}

\begin{abstract}
Two experiments were conducted to study the effects of $(\mathrm{Na}+\mathrm{K}) / \mathrm{Cl}$ ratio on the diet of commercial laying hens, at the end of egg production by the addition of sodium bicarbonate $\left(\mathrm{NaHCO}_{3}\right)$, sodium chloride $(\mathrm{NaCl})$, potassium chloride $(\mathrm{KCl})$ and ammonium chloride $\left(\mathrm{NH}_{4} \mathrm{Cl}\right)$, with regard to productive parameters and plasma blood characteristics. The first experiment involved five treatments: $0.30 \% \mathrm{NaCl} ; 0.30 \% \mathrm{NaCl}+0.95 \% \mathrm{NaHCO}_{3} ; 0.38 \% \mathrm{KCl}+0.67 \% \mathrm{NaHCO}_{3} ; 0.8 \% \mathrm{KCl}+1.62 \% \mathrm{NaHCO}$ and $0.38 \% \mathrm{KCl}$ $+2.56 \% \mathrm{NaHCO}_{3}$. The $(\mathrm{Na}+\mathrm{K}) / \mathrm{Cl}$ ratios were respectively, 3.46; 4.46; 4.46, 5.46 e 6.46. The second experiment involved four treatments: $0.30 \% \mathrm{NaCl} ; 0.47 \% \mathrm{NaHCO}_{3}+0.19 \mathrm{NH}_{4} \mathrm{Cl} ; 1.17 \% \mathrm{NaHCO}_{3}+0.19 \% \mathrm{NH}_{4} \mathrm{Cl}$ and $1.88 \% \mathrm{NaHCO}_{3}+0.19 \% \mathrm{NH}_{4} \mathrm{Cl}$. The $(\mathrm{Na}+\mathrm{K}) / \mathrm{Cl}$ ratios were $3.46 ; 4.46 ; 5.46$ and 6.46 , respectively. In the first experiment, the greatest feed intake was observed in the hens fed diet with $0.30 \% \mathrm{NaCl}$. The birds fed $\mathrm{KCl}$ presented better feed conversion. It was observed an increase in the plasma blood phosphorus and plasma blood $\mathrm{pH}$. The birds fed diets with 5.46 of $(\mathrm{Na}+\mathrm{K}) / \mathrm{Cl}$ ratio presented the highest plasma blood phosphorus. The hens receiving the diets with the 4.46 and $6.46(\mathrm{Na}+\mathrm{K}) / \mathrm{Cl}$ ratios presented an increase in the plasma blood $\mathrm{pH}$. In the second experiment, the egg weight decreased as $\mathrm{NaHCO}_{3}$ increased in the diet.
\end{abstract}

Key Words: blood, laying hens, performance, shell thickness

\section{Introdução}

Numerosos compostos têm sido adicionados ao alimento ou à água de bebida de poedeiras comerciais, com o objetivo de aliviar os efeitos adversos do estresse calórico. Muitos destes compostos são direcionados a corrigir o desequilíbrio ácido-base sangüíneo. $\mathrm{O}$ animal tem sua capacidade de dissipação de calor reduzida, quando é submetido à combinação de umidade relativa e temperatura acima da sua zona de conforto térmico, e, como conseqüência, há aumento da temperatura corporal, que, por sua vez, exerce impacto negativo sobre o desempenho do animal, afetando a eficiência alimentar, o consumo de alimento, a taxa de crescimento e a produtividade.

As aves estressadas pelo calor procuram restabelecer a temperatura interna com o aumento da frequência respiratória (LINSLEY e BURGER, 1964), o qual provoca queda nos níveis sangüíneos de $\mathrm{HCO}_{3}$ e $\mathrm{CO}_{2}$ e incremento do $\mathrm{pH}$, ocasionando

\footnotetext{
${ }^{1}$ Parte da Dissertação do segundo autor, para obtenção do grau de Mestre em Zootecnia.

2 Docente do Departamento de Zootecnia da FCAV/UNESP - Jaboticabal - SP.

${ }^{3}$ Docente da Faculdade de Agronomia - Paraguaçu Paulista - SP.

${ }^{4}$ Aluno do Curso de Pós-Graduação em Zootecnia da FCAV/UNESP - Jaboticabal - SP.
} 
alcalose respiratória. A alcalose induzida pelo estresse calórico tem sido relacionada ao elevado nível de corticosterona plasmático (BOWEN e WASHBURN, 1985) e ao balanço mineral negativo do potássio e do sódio (BELAY et al., 1980). Conseqüentemente, o $\mathrm{KCl}$ e o $\mathrm{NaCl}$, quando suplementados na água de bebida, podem reduzir a severidade do estresse calórico. TEETER et al. (1985) recomendaram o uso de $\mathrm{NH}_{4} \mathrm{Cl}$ como um acidificador sangüíneo potencial e o uso do bicabonato de sódio como uma fonte de dióxido de carbono para aliviar os efeitos da exposição crônica ao calor. A deposição do carbonato de cálcio para formar a casca do ovo é dependente do $\mathrm{pH}$ sangüíneo e é diminuída de maneira diretamente proporcional ao $\mathrm{pH}$, como resultado de alcalose respiratória (FRANK e BURGER, 1965). CHAN (1974) e MONGIN (1981) descreveram que o balanço alimentar de eletrólitos, particularmente o sódio, o potássio e o cloro, afeta o balanço ácido-base. $\mathrm{O}$ excesso de cloro na dieta diminui o pH sangüíneo e a concentração de íons de bicarbonato, a menos que o cloro seja balanceado por concentrações equivalentes de sódio ou potássio (AUSTIC, 1984).

Poucos relatos na literatura tratam da relação $(\mathrm{Na}+\mathrm{K}) / \mathrm{Cl}$ por meio da adição de $\mathrm{NH}_{4} \mathrm{Cl}$ em dietas de poedeiras comerciais. HALL e HELBACKA (1959) reportaram que, quando $\mathrm{NH}_{4} \mathrm{Cl}$ ou ácido clorídrico foi adicionado à dieta, houve aumento na altura do albúmen e concomitante redução na espessura da casca dos ovos. Resultados semelhantes foram obtidos por COMBS e HELBACKA (1960), que adicionaram $1,5 \%$ de $\mathrm{NH}_{4} \mathrm{Cl}$ a dietas de poedeiras comerciais.

O objetivo do presente trabalho foi estudar a relação $(\mathrm{Na}+\mathrm{K}) / \mathrm{Cl}$ da dieta de galinhas poedeiras comerciais, por itnermédio das adições de $\mathrm{NaHCO}_{3}$, $\mathrm{NaCl}, \mathrm{KCl}$ e $\mathrm{NH}_{4} \mathrm{Cl}$, sobre os parâmetros produtivos e constituintes sangüíneos de poedeiras comerciais em idade avançada.

\section{Material e Métodos}

Os experimentos foram conduzidos no Aviário Experimental da Granja Mizumoto (Assis - SP). O primeiro experimento foi realizado no período de $12 / 06 / 95$ a $31 / 07 / 95$, no qual se avaliou o efeito dos níveis e fontes de sódio, potássio e cloro sobre o desempenho e os componentes sangüíneos de poedeiras comerciais. Utilizaram-se 240 galinhas poedeiras da linhagem Hy-Line branca, com 54 semanas de idade, distribuídas em delineamento inteiramente casualizado envolvendo cinco tratamentos com quatro repetições de 12 aves cada, com duração de oito semanas. O primeiro tratamento consistiu da adição de $0.3 \%$ de $\mathrm{NaCl}$ e o segundo, na mesma percentagem de $\mathrm{NaCl}$ adicionado a $0,95 \%$ de $\mathrm{NaHCO}_{3}$. O terceiro, quarto e quinto tratamentos consistiram de uma dieta com 0,67 ; 1,62; e 2,56\% de $\mathrm{NaHCO}_{3}$, respectivamente, em adição a $0,38 \%$ de $\mathrm{KCl}$. Durante a realização do experimento, verificou-se diariamente a temperatura ambiente por intermédio de um termômetro, no qual se registraram os valores médios de 16,5 e $27,3^{\circ} \mathrm{C}$ para mínima e máxima.

Estabeleceu-se uma relação $(\mathrm{Na}+\mathrm{K}) / \mathrm{Cl}$ para os tratamentos que foram de 3,$46 ; 4,46 ; 4,46 ; 5,46 ; \mathrm{e}$ 6,46 , respectivamente. O cálculo da relação $(\mathrm{Na}+\mathrm{K}) / \mathrm{Cl}$ foi realizado tomando-se os valores de $\mathrm{Na}, \mathrm{K}$ e $\mathrm{Cl}$ fornecidos pela ração basal e pelos componentes utilizados $\left(\mathrm{NaHCO}_{3}, \mathrm{KCl}\right.$ e $\left.\mathrm{NaCl}\right)$. Amostras de sangue, de 15 aves por tratamento, foram colhidas da veia braquial na $8^{\mathrm{a}}$ semana do período experimental, tendo como critério de colheita uma hora após a realização da postura. $\mathrm{O}$ sangue foi centrifugado e o plasma, obtido, para posteriores determinações de cálcio, fósforo e pH sangüíneo.

No segundo experimento, realizado no período de 23/10 a 11/12/95, avaliou-se o efeito de fontes de sódio e cloro sobre o desempenho de poedeiras comerciais. Utilizaram-se 192 galinhas poedeiras da linhagem Hy-Line branca, com 54 semanas de idade, distribuídas em delineamento inteiramente casualizado envolvendo quatro tratamentos com quatro repetições de 12 aves cada, com duração de oito semanas. O primeiro tratamento consistiu da adição à dieta de $0,30 \%$ de $\mathrm{NaCl}$ e o segundo, terceiro e quarto tratamentos, da adição de 0,47 ; 1,17; e $1,88 \%$ de $\mathrm{NaHCO}_{3}$, respectivamente, em adição a $0,19 \%$ de $\mathrm{NH}_{4} \mathrm{Cl}$. Estabeleceu-se uma relação $(\mathrm{Na}+\mathrm{K}) / \mathrm{Cl}$ para os tratamentos de 3,46 ; 4,$46 ; 5,46$; e 6,46 respectivamente. Neste experimento, a temperatura variou entre 23,0 e $32,8^{\circ} \mathrm{C}$ (mínima e máxima).

Nos dois experimentos, as aves foram alojadas em gaiolas de arame em número de duas por gaiola. A água foi fornecida por intermédio de um sistema de fluxo contínuo, percorrendo toda a extensão frontal das gaiolas. As dietas basais (Tabela 1), foram formuladas para conter $17 \%$ de proteína bruta, $2800 \mathrm{kcal} \mathrm{EM} / \mathrm{kg}, 3,75 \%$ de cálcio e $0,63 \%$ de fósforo total, as quais foram fornecidas à vontade. 
Tabela 1 - Composição percentual das dietas experimentais Table 1 - Percentage composition of experimental diets

\begin{tabular}{|c|c|c|}
\hline & $\begin{array}{l}\text { Experimento } 1 \\
\text { Experiment } 1\end{array}$ & $\begin{array}{r}\text { Experimento } 2 \\
\text { Experiment } 2\end{array}$ \\
\hline Milho & 63,42 & 64,12 \\
\hline Corn & & \\
\hline Farelo de soja & 19,90 & 19,90 \\
\hline $\begin{array}{l}\text { Soybean meal } \\
\text { Farinha de carne e ossos } \\
\text { Bone and meat meal }\end{array}$ & 4,85 & 5,00 \\
\hline $\begin{array}{l}\text { Farinha de ostra } \\
\text { Oyster meal }\end{array}$ & 2,41 & 2,43 \\
\hline $\begin{array}{l}\text { Fosfato bicálcico } \\
\text { Dicalcium phosphate }\end{array}$ & 0,24 & 0,24 \\
\hline $\begin{array}{l}\text { Calcário calcítico } \\
\text { Limestone }\end{array}$ & 5,00 & 5,00 \\
\hline $\begin{array}{l}\text { Suplemento vit./mineral } \\
\text { Vitamin/mineral mix }\end{array}$ & 1,00 & 1,00 \\
\hline Óleo de soja & 0,24 & 0,24 \\
\hline $\begin{array}{l}\text { Soybean oil } \\
\text { Porção variável } \\
\text { Variable portion }\end{array}$ & $2,94^{1}$ & $2,07^{2}$ \\
\hline & \multicolumn{2}{|c|}{$\begin{array}{l}\text { Composição calculada } \\
\text { Calculated composition }\end{array}$} \\
\hline Proteína bruta (\%) & 17,00 & 17,00 \\
\hline $\begin{array}{l}\text { Crude protein } \\
\mathrm{EM}(\mathrm{kcal} / \mathrm{kg})\end{array}$ & 2800 & 2800 \\
\hline $\begin{array}{l}\text { Cálcio }(\%) \\
\text { Calcium }\end{array}$ & 3,75 & 3,75 \\
\hline $\begin{array}{l}\text { Fósforo total (\%) } \\
\text { Total phosphorus }\end{array}$ & 0,63 & 0,63 \\
\hline $\begin{array}{l}\text { Fósforo disponível (\%) } \\
\text { Available phosphorus }\end{array}$ & 0,45 & 0,45 \\
\hline $\begin{array}{l}\text { Metionina + cistina }(\%) \\
\text { Methionine + cystine }\end{array}$ & 0,62 & 0,62 \\
\hline $\begin{array}{l}\text { A porção variável constitui-se } \\
2 \text { A porção variável constitui-s } \\
\text { lavada. } \\
1 \text { The variable portion form of } \mathrm{NaC} C \\
2 \text { The variable portion form of } \mathrm{NaC}\end{array}$ & $\begin{array}{l}\text { e } \mathrm{NaCl}, \mathrm{KCl}, \mathrm{N} \\
\text { de } \mathrm{NaCl}, \mathrm{NH} \\
\mathrm{KCl} \mathrm{NaHCO}_{3} \text { ar }\end{array}$ & $\begin{array}{l}\mathrm{O}_{3} \text { e areia lavada } \\
\mathrm{NaHCO}_{3} \text { e areia } \\
\text { ashed sand. }\end{array}$ \\
\hline
\end{tabular}

A produção de ovos e o consumo de ração foram avaliados a intervalos de uma semana. A gravidade específica e a espessura da casca foram determinadas em todos os ovos produzidos nos dois últimos dias de cada semana, durante as oitos semanas de duração de cada experimento. O peso dos ovos foi determinado diariamente. Todos os dados foram submetidos à analise de variância, utilizando-se o teste de Duncan a $5 \%$ de probabilidade, como teste de comparação de médias.

\section{Resultados e Discussão}

Os efeitos dos diferentes níveis de sódio, cloro e potássio sobre o desempenho de poedeiras comerciais estão descritos na Tabela 2 . O balanço eletrolítico no organismo da ave pode ser afetado pelos níveis de eletrólitos da dieta e também pela produção endógena de ácidos. Como os eletrólitos são responsáveis pela manutenção da água corporal e do balanço iônico, as exigências de sódio, potássio e cloro não podem ser consideradas individualmente. O número de Mongin descreve a relação de eletrólitos na fórmula $(\mathrm{Na}+\mathrm{K})-\mathrm{Cl}$ expresso em $\mathrm{mEq} / \mathrm{kg}$ da dieta. No primeiro experimento, o número de Mongin do primeiro ao quinto tratamento foi $187,300,265,378,491$, respectivamente, não exercendo influência sobre os resultados encontrados. Observou-se que não houve diferença significativa para produção e massa de ovos, quando o $\mathrm{NaHCO}_{3}$ e o $\mathrm{KCl}$ foram adicionados à dieta, sendo que estes resultados concordam com os obtidos por MAKLED e CHARLES (1987) e GRIZZLE et al. (1992), que descrevem que a produção de ovos não é afetada pela adição de $\mathrm{NaHCO}_{3}$ à dieta.

$\mathrm{A}$ adição de $\mathrm{NaHCO}_{3}$ à dieta resultou em ovos significativamente mais leves $(\mathrm{P}<0,05)$, quando comparados àqueles cujas aves receberam somente o $\mathrm{NaCl}$ na ração. Comparando-se os tratamentos que continham $\mathrm{NaHCO}_{3}$ e $\mathrm{KCl}$, não se observaram diferenças estatísticas para este parâmetro. A relação $(\mathrm{Na}+\mathrm{K}) / \mathrm{Cl}$ igual a 3,46 resultou em ovos mais pesados, o que discorda dos relatos de HAMILTON e THOMPSON (1980), que conduziram experimentos semelhantes com rações que variaram de 0,40 a 7,69 na relação $(\mathrm{Na}+\mathrm{K}) / \mathrm{Cl}$ e não verificaram diferenças estatísticas para esta característica.

As aves que receberam dietas com adição de $\mathrm{NaCl}$ apresentaram maior consumo que as aves dos outros tratamentos, sendo que este resultado apresentou diferença estatística $(\mathrm{P}<0,05)$.

Os melhores resultados para conversão alimentar foram obtidos quando as aves receberam dietas com adição de $\mathrm{KCl}(\mathrm{P}<0,05)$, sendo que as aves que receberam dieta com $\mathrm{NaCl}$ apresentaram os piores resultados para este parâmetro.

A qualidade da casca dos ovos determinada pela gravidade específica e espessura da casca não foi 
JUNQUEIRA et al.

Tabela 2 - Efeitos dos níveis e fontes de sódio, cloro e potássio sobre a produção de ovos (PrO), peso de ovos (PO), consumo de ração (CR), conversão alimentar (CA) e massa de ovos (MO) de poedeiras comerciais (Experimento 1)

Table 2 - Effects of levels and sources of sodium, chlorine and potassium on the egg production (PrO), egg weight (PO), feed intake (CR), feed/egg ratio (CA) and egg mass (MO) of laying hens (Experiment 1)

\begin{tabular}{|c|c|c|c|c|c|c|c|c|c|c|}
\hline \multirow{2}{*}{$\begin{array}{l}\text { Tratamento }{ }^{1} \\
\text { Treatment }\end{array}$} & $\mathrm{Na}$ & $\mathrm{Cl}$ & $\mathrm{K}$ & \multirow{2}{*}{$\begin{array}{c}\text { Relação } \\
\text { Ratio } \\
(\mathrm{Na}+\mathrm{K}) / \mathrm{Cl}\end{array}$} & \multirow{2}{*}{$\begin{array}{l}\text { Mongin } \\
\mathrm{mEq} / \mathrm{kg}\end{array}$} & \multirow{2}{*}{$\begin{array}{c}\mathrm{PrO}^{2} \\
(\%)\end{array}$} & \multirow{2}{*}{$\begin{array}{l}\text { PO } \\
(\mathrm{g})\end{array}$} & \multirow{2}{*}{$\begin{array}{l}\text { CR } \\
(\mathrm{g})\end{array}$} & \multirow{2}{*}{$\begin{array}{c}\mathrm{CA} \\
(\mathrm{kg} / \mathrm{dz})\end{array}$} & \multirow{2}{*}{$\begin{array}{l}\mathrm{MO} \\
(\mathrm{g})\end{array}$} \\
\hline & & $(\%)$ & & & & & & & & \\
\hline$\overline{1}$ & 0,17 & 0,26 & 0,73 & 3,46 & 187 & $73,85^{\mathrm{a}}$ & $62,93^{\mathrm{a}}$ & $107,56^{\mathrm{a}}$ & $2,29^{\mathrm{a}}$ & $46,5^{\mathrm{a}}$ \\
\hline 2 & 0,43 & 0,26 & 0,73 & 4,46 & 300 & $75,30^{\mathrm{a}}$ & $61,36^{\mathrm{b}}$ & $100,15^{b}$ & $2,19^{\mathrm{ab}}$ & $46,2^{\mathrm{a}}$ \\
\hline 3 & 0,23 & 0,26 & 0,93 & 4,46 & 265 & $77,05^{\mathrm{a}}$ & $61,58^{\mathrm{b}}$ & $95,54^{\mathrm{c}}$ & $2,08^{b}$ & $47,5^{\mathrm{a}}$ \\
\hline 4 & 0,49 & 0,26 & 0,93 & 5,46 & 378 & $74,00^{\mathrm{a}}$ & $61,89^{\mathrm{b}}$ & $95,54^{\mathrm{c}}$ & $2,07^{\mathrm{b}}$ & $45,8^{\mathrm{a}}$ \\
\hline 5 & 0,75 & 0,26 & 0,93 & 6,46 & 491 & $72,36^{\mathrm{a}}$ & $62,04^{\mathrm{b}}$ & $95,91^{\mathrm{c}}$ & $2,07^{\mathrm{b}}$ & $44,9^{\mathrm{a}}$ \\
\hline $\mathrm{CV}(\%)$ & & & & & & 4,3 & 0,9 & 2,2 & 2,5 & 4,3 \\
\hline
\end{tabular}

1 Tratamentos (Treatments): $1-0,30 \% \mathrm{NaCl} ; 2-0,30 \% \mathrm{NaCl}+0,95 \% \mathrm{NaHCO}_{3} ; 3-0,67 \% \mathrm{NaHCO}_{3}+0,38 \% \mathrm{KCl} ; 4-1,62 \% \mathrm{NaHCO}_{3}$ $+0,38 \% \mathrm{KCl} ; 5-2,56 \% \mathrm{NaHCO}_{3}+0,38 \% \mathrm{KCl}$.

2 a, b, c DUNCAN $(\mathrm{P}<0,05)$.

Tabela 3 - Efeitos dos níveis e fontes de sódio, cloro e potássio sobre a qualidade da cascas dos ovos (Experimento 1)

Table 3 - Effects of levels and sources of sodium, chlorine and potassium on the egg shell quality (Experiment 1)

\begin{tabular}{|c|c|c|c|c|c|c|c|}
\hline $\begin{array}{l}\text { Tratamento } \\
\text { Treatment }\end{array}$ & $\mathrm{Na}$ & $(\%)$ & K & $\begin{array}{c}\text { Relação } \\
\text { Ratio } \\
(\mathrm{Na}+\mathrm{K}) / \mathrm{Cl}\end{array}$ & $\begin{array}{l}\text { Mongin } \\
\mathrm{mEq} / \mathrm{kg}\end{array}$ & $\begin{array}{c}\text { Gravidade } \\
\text { específica }{ }^{2} \\
\text { Specific } \\
\text { gravity }\end{array}$ & $\begin{array}{c}\text { Espessura } \\
\text { da casca }(\mathrm{mm}) \\
\text { Shell } \\
\text { thickness }\end{array}$ \\
\hline 1 & 0,17 & 0,26 & 0,73 & 3,46 & 187 & $1,085^{\mathrm{a}}$ & $0,35^{\mathrm{a}}$ \\
\hline 2 & 0,43 & 0,26 & 0,73 & 4,46 & 300 & $1,084^{\mathrm{a}}$ & $0,34^{\mathrm{a}}$ \\
\hline 3 & 0,23 & 0,26 & 0,93 & 4,46 & 265 & $1,085^{\mathrm{a}}$ & $0,34^{\mathrm{a}}$ \\
\hline 4 & 0,49 & 0,26 & 0,93 & 5,46 & 378 & $1,083^{\mathrm{a}}$ & $0,34^{\mathrm{a}}$ \\
\hline 5 & 0,75 & 0,26 & 0,93 & 6,46 & 491 & $1,085^{\mathrm{a}}$ & $0,35^{\mathrm{a}}$ \\
\hline $\mathrm{CV}(\%)$ & & & & & & 0,2 & 1,1 \\
\hline
\end{tabular}

1 Tratamentos (Treatments): $1-0,30 \% \mathrm{NaCl} ; 2-0,30 \% \mathrm{NaCl}+0,95 \% \mathrm{NaHCO}_{3} ; 3-0,67 \% \mathrm{NaHCO}_{3}+0,38 \% \mathrm{KCl} ; 4-1,62 \%$ $\mathrm{NaHCO}_{3}+0,38 \% \mathrm{KCl} ; 5-2,56 \% \mathrm{NaHCO}_{3}+0,38 \% \mathrm{KCl}$.

2 a, DUNCAN $(P>0,05)$.

afetada pela relação $(\mathrm{Na}+\mathrm{K}) / \mathrm{Cl}$ (Tabela 3). Estes resultados discordam de CHARLES et al. (1972) e MAKLED e CHARLES (1987), que descreveram efeitos benéficos da adição de 0,25 ou $0,50 \%$ de $\mathrm{NaHCO}_{3}$ em rações de poedeiras, as quais produziram ovos com cascas mais espessas. MILES e HARMS (1982) registraram que a adição de $\mathrm{NaHCO}_{3}$ às dietas de poedeiras proporcionou significativa melhora na taxa de produção e qualidade da casca dos ovos, quando observada a gravidade específica e espessura da casca.

Os resultados obtidos para níveis plasmáticos de cálcio, fósforo e pH sangüíneo são demonstrados na Tabela 4. Não houve diferenças estatísticas para os níveis de cálcio plasmático. Contudo, ocorreu aumento do cálcio plasmático das aves que receberam as dietas contendo $\mathrm{NaHCO}_{3}$ e $\mathrm{KCl}$. HODGES e
LORCHER (1967) investigaram o destino do carbono oriundo do bicarbonato em dietas de galinhas poedeiras, tendo reportado que o bicarbonato no sistema circulatório não se constitui no maior precursor do carbonato da casca dos ovos, sugerindo, portanto, que a célula uterina seja a responsável pela formação do íon carbonato, o qual se incorpora ao cálcio para formar o carbonato de cálcio. Observou-se significativa variação nos valores de fósforo plasmático e $\mathrm{pH}$ sangüíneo das aves que receberam os diferentes tratamentos $(\mathrm{P}<0,05)$, nos quais as aves que receberam $\mathrm{KCl}$ adicionado à dieta apresentaram maior concentração plasmática de fósforo, sendo que o $\mathrm{pH}$ sangüíneo foi maior naquelas que receberam as dietas com $\mathrm{NaCl}+\mathrm{NaHCO}_{3}$ e o nível mais alto de $\mathrm{KCl}$. MILES (1980) relata que o sódio da dieta, ao ser 
1114 Rev. bras. zootec.

absorvido, se combina com o fósforo plasmático, dando formação ao fosfato de sódio, e, por conseguinte, facilita sua eliminação pelos rins. Dessa forma, a diminuição do fósforo plasmático traz efeitos benéficos sobre a incorporação do cálcio ao íon carbonato, com conseqüente elevação da síntese de carbonato de cálcio. MOGIN e SAUVEUR (1979) e MILES et al. (1984) verificaram que o fósforo plasmático das poedeiras começa a se declinar do momento em que ocorre a postura até 6 horas após a oviposição e, então, novamente se eleva.

Na Tabela 5, são apresentadas as características produtivas das poedeiras comerciais, no segundo experimento. Não houve diferença significativa ( $\mathrm{P}>0,05)$ na produção de ovos e no consumo de alimentos por parte das poedeiras alimentadas com rações contendo as diferentes relações $(\mathrm{Na}+\mathrm{K}) / \mathrm{Cl}$. Estes resultados concordam com os obtidos por HUNT e AITKEN (1962), os quais relataram que a adição de $\mathrm{NH}_{4} \mathrm{Cl}$ não afetou a taxa de produção de ovos. $\mathrm{O}$ peso médio dos ovos foi afetado significativamente, tendo-se observado que os mais pesados foram obtidos pelas aves que consumiram rações contendo
$0,30 \%$ de $\mathrm{NaCl}$ e $0,47 \%$ de $\mathrm{NaHCO}_{3}$ mais $0,19 \%$ de $\mathrm{NH}_{4} \mathrm{Cl}$, respectivamente, nas relações $(\mathrm{Na}+\mathrm{K}) / \mathrm{Cl}$ iguais a 3,46 e 4,46 . Verificou-se que houve diminuição do peso dos ovos, à medida que se elevou a relação $(\mathrm{Na}+\mathrm{K}) / \mathrm{Cl}$, com a adição de $\mathrm{NaHCO}_{3}$, o que discorda dos relatos de HAMILTON e THOMPSON (1980), os quais verificaram que a relação $(\mathrm{Na}+\mathrm{K}) / \mathrm{Cl}$ não exerceu qualquer efeito sobre o parâmetro analisado. As médias obtidas para massa de ovos não apresentaram diferença estatística.

A gravidade específica e a espessura da casca (Tabela 6) não sofreram efeito benéfico da adição de $\mathrm{NaHCO}_{3}$ e $\mathrm{NH}_{4} \mathrm{Cl}$. No entanto, HELBACKA e HALL (1958) e HALL e HELBACKA (1959) observaram que a adição de $\mathrm{NH}_{4} \mathrm{Cl}$ à ração diminuiu a espessura da casca dos ovos, sem, contudo, afetar a altura do albúmen. Da mesma forma, HUNT e AITKEN (1962 a, b) relataram que a gravidade específica dos ovos foi significativamente reduzida, quando se adicionaram $2 \%$ de $\mathrm{NH}_{4} \mathrm{Cl}$ à dieta de poedeiras comerciais. Para os quatro tratamentos, observaram-se, respectivamente, os valores de 187 , 200, 287 e 374 para o número de Mongin (mEq/kg).

Tabela 4 - Efeitos dos níveis e fontes de sódio, cloro e potássio sobre a concentração plasmática de cálcio e fósforo e pH sangüíneo de poedeiras comerciais (Experimento 1)

Table 4 - Effects of levels and sources of sodium, chlorine and potassium on concentration plasma of calcium and phosphorus and $\mathrm{pH}$ of blood of laying hens (Experiment 1)

\begin{tabular}{|c|c|c|c|c|c|c|c|c|}
\hline \multirow{2}{*}{$\begin{array}{l}\text { Tratamento } \\
\text { Treatment }\end{array}$} & $\mathrm{Na}$ & $(\%)$ & $\mathrm{K}$ & \multirow[t]{2}{*}{$\begin{array}{c}\text { Relação } \\
\text { Ratio } \\
(\mathrm{Na}+\mathrm{K}) / \mathrm{Cl}\end{array}$} & \multirow[t]{2}{*}{$\begin{array}{l}\text { Mongin } \\
\mathrm{mEq} / \mathrm{kg}\end{array}$} & \multicolumn{2}{|c|}{$\begin{array}{c}\text { Plasma }^{2} \\
\text { Plasma } \\
(\mathrm{mg} / 100 \mathrm{~mL})\end{array}$} & \multirow{2}{*}{$\begin{array}{c}\mathrm{pH} \\
\text { sangüíneo } \\
\text { Blood } \\
p H\end{array}$} \\
\hline & & $(\%)$ & & & & $\mathrm{Ca}$ & $\mathrm{P}$ & \\
\hline 1 & 0,17 & 0,26 & 0,73 & 3,46 & 187 & $27,07^{\mathrm{a}}$ & $6,37^{\mathrm{b}}$ & $7,32^{\mathrm{cd}}$ \\
\hline 2 & 0,43 & 0,26 & 0,73 & 4,46 & 300 & $27,05^{\mathrm{a}}$ & $6,51^{\mathrm{b}}$ & $7,44^{\mathrm{ab}}$ \\
\hline 3 & 0,23 & 0,26 & 0,93 & 4,46 & 265 & $27,15^{\mathrm{a}}$ & $7,14^{\mathrm{ab}}$ & $7,27^{\mathrm{d}}$ \\
\hline 4 & 0,49 & 0,26 & 0,93 & 5,46 & 378 & $27,23^{\mathrm{a}}$ & $7,37^{\mathrm{a}}$ & $7,37^{b c}$ \\
\hline 5 & 0,75 & 0,26 & 0,93 & 6,46 & 491 & $28,33^{\mathrm{a}}$ & $7,02^{\mathrm{ab}}$ & $7,48^{\mathrm{a}}$ \\
\hline $\mathrm{CV}(\%)$ & & & & & & 8,5 & 13,2 & 1,4 \\
\hline
\end{tabular}

1 Tratamentos (Treatments): $1-0,30 \% \mathrm{NaCl} ; 2-0,30 \% \mathrm{NaCl}+0,95 \% \mathrm{NaHCO}_{3} ; 3-0,67 \% \mathrm{NaHCO}_{3}+0,38 \% \mathrm{KCl} ; 4-1,62 \%$ $\mathrm{NaHCO}_{3}+0,38 \% \mathrm{KCl} ; 5-2,56 \% \mathrm{NaHCO}_{3}+0,38 \% \mathrm{KCl}$.

2 a, b, c, d DUNCAN $(P<0,05)$. 
JUNQUEIRA et al.

Tabela 5 - Efeitos dos níveis e fontes de sódio e cloro sobre a produção de ovos (PrO), o peso de ovos (PO), o consumo de ração (CR), a conversão alimentar (CA) e a massa de ovos (MO) de poedeiras comerciais (Experimento 2)

Table 5 - Effects of levels and sources of sodium and chlorine on the egg production (PrO), egg weight (PO), feed intake (CR), feed/egg ratio (CA) and egg mass (MO) of laying hens (Experiment 2)

\begin{tabular}{|c|c|c|c|c|c|c|c|c|c|c|}
\hline & $\mathrm{Na}$ & $\mathrm{Cl}$ & $\mathrm{K}$ & Relação & Mongin & $\mathrm{PrO}^{2}$ & $\mathrm{PO}$ & $\mathrm{CR}$ & $\mathrm{CA}$ & MO \\
\hline $\begin{array}{l}\text { Tratamento }^{1} \\
\text { Treatment }\end{array}$ & & $(\%)$ & & $\begin{array}{c}(\mathrm{Na}+\mathrm{K}) / \\
\mathrm{Cl}\end{array}$ & $\mathrm{mEq} / \mathrm{kg}$ & $(\%)$ & (g) & (g) & $(\mathrm{kg} / \mathrm{dz})$ & (g) \\
\hline 1 & 0,17 & 0,26 & 0,73 & 3,46 & 187 & $75,19^{\mathrm{a}}$ & $64,54^{\mathrm{ab}}$ & $101,56^{\mathrm{a}}$ & $2,1^{\mathrm{a}}$ & $48,52^{\mathrm{a}}$ \\
\hline 2 & 0,16 & 0,20 & 0,73 & 4,46 & 200 & $72,73^{\mathrm{a}}$ & $64,98^{\mathrm{a}}$ & $102,46^{\mathrm{a}}$ & $2,1^{\mathrm{a}}$ & $47,26^{\mathrm{a}}$ \\
\hline 3 & 0,36 & 0,20 & 0,73 & 5,46 & 287 & $74,33^{\mathrm{a}}$ & $64,15^{\mathrm{b}}$ & $101,75^{\mathrm{a}}$ & $2,1^{\mathrm{a}}$ & $47,68^{a}$ \\
\hline 4 & 0,56 & 0,20 & 0,73 & 6,46 & 374 & $71,88^{\mathrm{a}}$ & $63,29^{c}$ & $99,52^{\mathrm{a}}$ & $2,1^{\mathrm{a}}$ & $45,49^{\mathrm{a}}$ \\
\hline $\mathrm{CV}(\%)$ & & & & & & 5,5 & 0,5 & 4,2 & 4,5 & 5,5 \\
\hline
\end{tabular}

1 Tratamentos (Treatments): $1-0,30 \% \mathrm{NaCl} ; 2-0,47 \% \mathrm{NaHCO}_{3}+0,19 \% \mathrm{NH}_{4} \mathrm{Cl} ; 3-1,17 \% \mathrm{NaHCO}_{3}+0,19 \% \mathrm{NH}_{4} \mathrm{Cl} ; 4-1,88 \%$ $\mathrm{NaHCO}_{3}+0,19 \% \mathrm{NH}_{4} \mathrm{Cl}$.

2 a, b, c $(P<0,05)$.

Tabela 6 - Efeitos dos níveis e fontes de sódio e cloro sobre a qualidade da casca de ovos (Experimento 2) Table 6 - Effects of levels and sources of sodium and chlorine on egg shell quality (Experiment 2)

\begin{tabular}{|c|c|c|c|c|c|c|c|}
\hline $\begin{array}{l}\text { Tratamento }^{1} \\
\text { Treatment }\end{array}$ & $\mathrm{Na}$ & $(\%)$ & $\mathrm{K}$ & $\begin{array}{c}\text { Relação } \\
\text { Ratio } \\
(\mathrm{Na}+\mathrm{K}) / \mathrm{Cl}\end{array}$ & $\begin{array}{l}\text { Mongin } \\
\mathrm{mEq} / \mathrm{kg}\end{array}$ & $\begin{array}{c}\text { Gravidade } \\
\text { Específica }^{2} \\
\text { Specific } \\
\text { Gravity }\end{array}$ & $\begin{array}{c}\text { Espessura } \\
\text { da casca }(\mathrm{mm}) \\
\text { Shell } \\
\text { thickness }\end{array}$ \\
\hline 1 & 0,17 & 0,26 & 0,73 & 3,46 & 187 & $1,085^{\mathrm{a}}$ & $0,35^{\mathrm{a}}$ \\
\hline 2 & 0,16 & 0,20 & 0,73 & 4,46 & 200 & $1,084^{\mathrm{a}}$ & $0,35^{\mathrm{a}}$ \\
\hline 3 & 0,36 & 0,20 & 0,73 & 5,46 & 287 & $1,083^{\mathrm{a}}$ & $0,34^{\mathrm{a}}$ \\
\hline 4 & 0,56 & 0,20 & 0,73 & 6,46 & 374 & $1,084^{\mathrm{a}}$ & $0,35^{\mathrm{a}}$ \\
\hline$\overline{\mathrm{CV}(\%)}$ & & & & & & 0,1 & 1,4 \\
\hline
\end{tabular}

\section{Conclusões}

Nos níveis estudados, as adições de cloreto de sódio, cloreto de potássio, cloreto de amônia e bicarbonato de sódio não interferiram na qualidade da casca de ovos, influenciando, porém, o desempenho e os parâmetros sangüíneos das poedeiras às 54 semanas de idade. Ocorreu melhor produção de ovos com a relação $(\mathrm{Na}+\mathrm{K}) / \mathrm{Cl}$ de 4,46 . A adição de cloreto de potássio, juntamente com o bicarbonato de sódio, diminuiu o consumo de ração e melhorou a conversão alimentar das aves, aumentando também a concentração do fósforo plasmático.

\section{Referências Bibliográficas}

AUSTIC, R.E. 1984. Excess dietary chloride depresses eggshell quality. Poult. Sci., 63(9):1773-1777.

BELAY, T., WIERNUSZ, C.J., TEETER, R.G. 1980. Mineral balance of heat distressed broilers. Oklahoma: Oklahoma Agricultural Experiment Station. p.189-194.

BOWEN, S.J., WASHBURN, K.W. 1985. Thyroid and adrenal response to heat stress in chickens and quail differing in heat tolerance. Poult. Sci., 64(1):149-154.

CHAN, J.C.M. 1974. The influence of dietary intake on endogenous acid production. Theoretical and experimental background. Nutrition Metabolism, 16(1):1-9.

CHARLES, O.W., CLARK, R., HUSTON, T.M. et al. 1972. The effect of calcium source, sodium bicarbonate and temperature on egg shell quality. Poult. Sci., 51(5):1793 (Abstract).

COMBS, G.F., HELBACKA, N.V. 1960. Studies with laying 
1116 Rev. bras. zootec.

hens. 1 - Effect of dietary fat, protein levels and other variables in practical rations. Poult. Sci., 39(1):271-279.

FRANK, F.R., BURGER, R.E. 1965. The effect of carbon dioxide inhalation and sodium bicarbonate ingestion on eggshell deposition. Poult. Sci., 44(6):1604-1606.

GRIZZLE, J., IHEANACHO, M., SAXTON, A. et al. 1992. Nutritional and environmental factors involved in egg shell quality of laying hens. Br. Poult. Sci., 33(4):781-784.

HALL, K.N., HELBACKA, N.V. 1959. Improving albumen quality. Poult. Sci., 38(1):111-114.

HAMILTON, R.M.G., THOMPSON, B.K. 1980. Effects of sodium plus potassium to chloride ratio in pratical - type diets on blood gas levels in three strains of White Leghorn hens and the relatioship between acid-base balance and egg shell strenth. Poult. Sci., 59(6):1294-1303.

HELBACKA, N.V., HALL, H.N. 1958. Characteristics of albumen and shell quality of eggs from layers fed $\mathrm{NH}_{4} \mathrm{Cl}$ in the diet. Poult. Sci., 37(5):1211.

HODGES, R.D., LORCHER, K. 1967. Possible sources of the carbonate fraction of egg shell calcium carbonate. Nature, 216(5094):609-610.

HUNT, J.R., AITKEN, J.R. 1962. Studies of influence of ascorbic acid on shell quality. Poult. Sci., 41(1):219-226.

LINSLEY, J.G., BURGER, R.E. 1964. Respiratory and cardiovascular response in the hyperthermic domestic cock. Poult. Sci., 43(1):291-305.

MAKLED, M.N., CHARLES, O.W. 1987. Eggshell quality as influenced by sodium bicarbonate, calcium source and photoperiod. Poult. Sci., 66(4):705-712.
MILES, R.D., HARMS, R.H. 1982. Relationship between egg specific gravity and plasma phosphorus form hens fed different dietary calcium, phosphorus and sodium levels. Poult. Sci., 61(1):175-177.

MILES, R.D. The role of phosphorus in egg shell quality. In: THE FLORIDA NUTRITION CONFERENCE, 1980. Proceedings... University of Florida, 1980. p.95-110.

MILES, R.D., JUNQUEIRA, O.M., HARMS, R.H. 1984. Plasma phosphorus at 0,6 and 21 hours post oviposition in hens laying in the morning or the afternoon. Poult. Sci., 63(2):354-359.

MONGIN, P., SAUVEUR, B. 1979. Plasma inorganic phosphorus concentration during egg-shell formation. Effect of the physical form of the dietary calcium. Br. Poult. Sci., 20(2):401-412.

MONGIN, P. 1981. Recent advances in dietary anion-cation balance: applications in poultry. Proceedings Nutrition Society, 40:285-294.

TEETER, R.G., SMITH, M.O., OWENS, F.N. et al. 1985. Chronic heat stress and respiratory alkalosis: occurrence and treatment in broiler chickens. Poult. Sci., 64(6):1060-1064.

Recebido em: 28/07/1999

Aceito em: 03/01/2000 